\title{
Human-Machine Cooperative Decision Making Outperforms Individualism and Autonomy
}

This paper was downloaded from TechRxiv (https://www.techrxiv.org).

\section{LICENSE}

CC BY-NC-SA 4.0

SUBMISSION DATE / POSTED DATE

$10-10-2021 / 18-10-2021$

\section{CITATION}

Rothfuß, Simon; Wörner, Maximilian; Inga, Jairo; Kiesel, Andrea; Hohmann, Sören (2021): Human-Machine Cooperative Decision Making Outperforms Individualism and Autonomy. TechRxiv. Preprint. https://doi.org/10.36227/techrxiv.16780273.v1

DOI

10.36227/techrxiv.16780273.v1 


\title{
Human-Machine Cooperative Decision Making Outperforms Individualism and Autonomy
}

\author{
Simon Rothfuß $\beta^{1}$, IEEE Member, Maximilian Wörner ${ }^{1}$, Jairo Inga ${ }^{1}$, \\ Andrea Kiesel $^{2}$ and Sören Hohmann ${ }^{1}$, IEEE Member
}

\begin{abstract}
The experiment reported in this paper provides a first experimental evaluation of human-machine cooperation on decision level: It explicitly focuses on the interaction of human and machine in cooperative decision making situations for which a suitable experimental design is introduced. Furthermore, it challenges conventional leader-follower approaches by comparing them to newly proposed automation designs based on cooperative decision making models. These models originate from negotiation theory and game theory and allow for an investigation of cooperative decision making between equal partners. This equality is motivated by similar approaches on the action level of humanmachine cooperation.

The experiment's results indicate an added value of the proposed automation designs in terms of objective cooperative performance as well as human trust in and satisfaction with the cooperation. Hence, the experiment yields the same insight on decision level as already observed on action level: it may be beneficial to design machines as equal cooperation partners and in accordance to models of emancipated human-machine cooperation.
\end{abstract}

Index Terms-Human-Automation Interaction, Decision Making, Human-Vehicle Interface, Cognitive Systems Engineering.

\section{INTRODUCTION}

$\mathbf{I}$ $\mathrm{N}$ the past decade, the interaction and mutual beneficial coexistence of humans and machines has seen a major research boost in various domains such as medical and systems engineering and ergonomics. Currently, the first concepts find their way into application in robotics, medical technologies, advanced driving assistance systems etc. [1], [2]. Encouraged by this success, this paper focuses on innovative work of model-based automation design in the context for humanmachine cooperation [3]-[7]. In particular, it provides some evidence for synergies of human-machine cooperation on higher levels than currently available and investigated.

\section{A. The Next Level of Human-Machine Cooperation}

Literature in the context of human-machine cooperation offers a great variety of concepts to describe human-machine interaction and corresponding automation design approaches such as e.g. shared control and human-machine cooperation

This work has been submitted to the IEEE for possible publication. Copyright may be transferred without notice, after which this version may no longer be accessible.

${ }^{1}$ Simon Rothfuß, Maximilian Wörner, Jairo Inga and Sören Hohmann are with the Institute of Control Systems, Karlsruhe Institute of Technology, Karlsruhe, Germany. Contact information at www.irs.kit.edu

${ }^{2}$ Andrea Kiesel is with the Department of Psychology at the University of Freiburg, Freiburg, Germany. kieselepsychologie.uni-freiburg.de

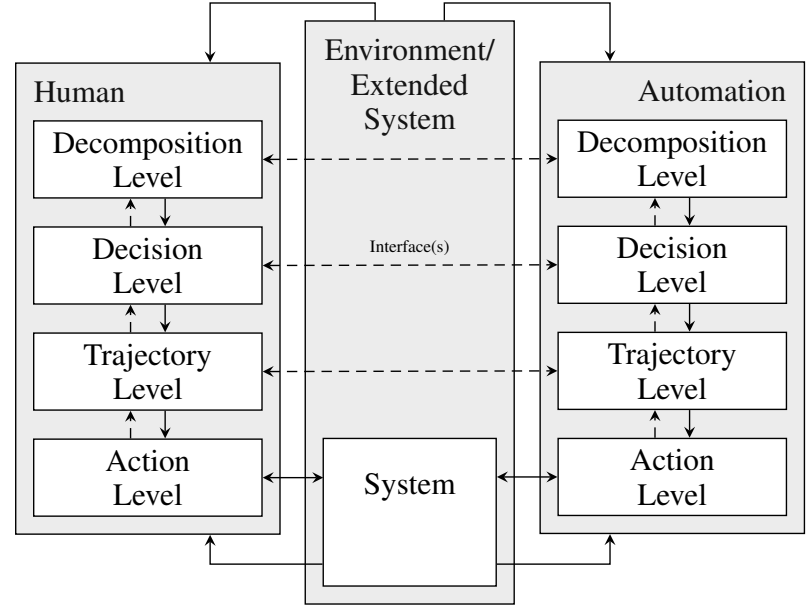

Fig. 1. The butterfly model for human-machine cooperation adapted from [6] and inspired by [1], [8].

[8]. Unfortunately, there also exists a heterogeneous set of definitions and naming of these often similar concepts, e. g. [1] vs. [8]. However, one common approach for the classification of human-machine cooperation are hierarchical layer models [1], [8]-[10] oriented on human cognition models (e. g. [11]). Fig. 1 shows such a layer model which is adapted from [6]. Interaction of human and machine is possible and can be found across all levels of human-machine cooperation [1]. In fact, Flemisch et al. [8] recently pointed to the necessity to consider all levels of the human-machine cooperation to fully exploit its benefits.

However, existing automation design approaches for humanmachine cooperation are mostly found on the trajectory and action levels of the cooperation, where human and machine interact physically close and on a control trajectory basis, providing evidence that cooperation and shared control have benefits compared to individualism (i. e. individual human performance) and autonomy [2], [12]-[15]. Despite these results, similar investigations for the next higher level of decision making have not been made. On this level, the leader-follower paradigm with various degrees of dynamic assignments of the leader and follower role is widely applied, leaving an assistive character for the automation [12], [13], [16], [17]. To the best of our knowledge, there is no cooperative system design available on the decision level that considers human and machine as equal, emancipated cooperation partners. This investigative and methodical gap in research is addressed in this paper, motivated by the success of emancipated human- 
machine cooperation on lower levels and by an increased degree of autonomy in various application domains [18], [19], i. e. human action may no longer be required on the lower levels of human-machine cooperation.

An example of this research gap can be observed in highly automated driving: Assume that the vehicle autonomously performs the control tasks of driving and human-machine cooperation is only required for aspects of navigation and guidance. If e. g. the vehicle is controlled on a maneuver basis, the choice of the maneuver depends on the information on current and future traffic. In this scenario, local traffic perception of humans may outperform the vehicle's perception. The opposite could be assumed for future traffic influences. Hence, the objective of human-machine cooperation in this context is to intuitively combine the information sets of human and automation which requires an emancipated interaction and thus improve the joint choice of the driving maneuver.

\section{B. Our Approach}

In order to establish human-machine cooperation on decision level we choose to design the automation by means of a behavioral model of cooperation partners in a cooperative decision making scenario. This approach is motivated by the success of model-based automation designs on the action level of human-machine cooperation [2]. Consequently, we proposed two models of cooperative decision making in previous papers: the Adaptive Negotiation Framework [4] and the $n$-Stage War of Attrition [7]. The two models originate from negotiation theory and game theory and provide means to describe cooperative decision making among machines and humans, respectively: negotiation theory is based on automated decision makers that are required to explicitly agree on a conflict resolution whereas game theory comprises interaction models of individual, selfish decision makers, e. g. humans and animals. Hence, these two models close the gap between the two extremes of automation-centered and human-centered decision making approaches which conventionally led to leaderfollower designs.

Analogously to existing cooperative approaches on action level, the new models' key feature is to recognize both partners of the cooperation, human and machine, as emancipated equal participants of the decision making in question. Naturally, this requires that both cooperation partners are more or less equally performant regarding situation awareness, judgement and communication abilities [1].

After introducing the cooperative decision making models, we were able to demonstrate in our previous work that both are valid to model human-like concession behavior in humanmachine cooperative decision making scenarios, cf. [6]. Furthermore, we established corresponding automation designs enabling human-machine cooperative decision making. In Section II, we briefly present both proposed models of cooperative decision making and the corresponding automation designs.

\section{Our Contribution: Research Questions and Hypotheses}

Having introduced suitable models of human-machine cooperative decision making and corresponding automation de- signs, the remaining research questions and therefore this paper's focus are:

R1: Are the proposed automation designs based on cooperative decision making models beneficial compared to leader-follower-based automation designs in terms of objective cooperation performance and subjective assessment?

R2: How to suitably design an experiment to answer R1?

Hence, this paper's first major contribution is our approach to the latter question: we propose a controlled experimental design (cf. Section III) focusing explicitly on the humanmachine interaction on decision level while creating a realistic scenario for the participants. To be more specific, we deploy a driving simulator of a highly automated vehicle putting the participants in a dynamic and realistic driving scenario in which they have to interact with the vehicle's automation in order to cooperatively decide on driving maneuvers. By means of an adequate interface design for cooperative decision making, we aim to limit the potentially disturbing influence of other factors of human-machine interaction such as e.g. inequality of physical strength. Furthermore, our experimental setting allows for a more general evaluation scope of the studied human-machine cooperation on decision level, i. e. for defining an objective measure of cooperative performance in addition to the usually applied subjective or individual measures in human-machine experiments.

Based on this new experimental design, the second major contribution of this paper is the evaluation of R1 in Section IV and V. In detail, we compare the two automation designs based on the new cooperative decision making models (the n-stage war of attrition and the adaptive negotiation model) with the two leader-follower-based automation designs (human in lead while the automation follows, and vice versa). The comparison's evaluation is conducted with respect to objective measures and subjective assessment and investigates the following hypotheses:

H1: The objective performance of the human-machine cooperation on decision level with automation designs based on cooperative decision making models is significantly better compared to the state-of-the-art leader-followerbased automation designs.

$\mathrm{H} 2$ : The participants' subjective assessments are significantly better for the proposed automation designs based on cooperative decision making models than for the stateof-the-art leader-follower-based automation designs in terms of satisfaction and trust in the cooperation as well as intuition of interaction. The opposite is expected regarding the transparency of interaction.

The remaining paper is structured as follows: Section II briefly introduces the proposed models for human-machine cooperative decision making and the subsequent automation designs. The experimental design and evaluation results of the automation designs' comparison are provided in Section III and IV, respectively. This is followed by the discussion of the results in Section V and an overall conclusion in Section VI. 


\section{Methods: Automation Design Based on INTRODUCED MODELS FOR HUMAN-MACHINE COOPERATIVE DECISION MAKING}

In this section, we briefly explain two models of humanmachine cooperative decision making that originate from negotiation theory and game theory. They are suitable to model human-like concession behavior in human-machine cooperative decision making scenarios, cf. [6]. Motivated by the success of human-like automation design on the action/ operational level of human-machine cooperation, we apply these models of cooperative decision making to design the automation on decision level to exhibit human-like behavior. Lastly, we elaborate on the automation design in case of conventional leader-follower approaches allowing for the subsequent experimental evaluation and comparison of these approaches with the automation design based on models of human-machine cooperative decision making.

\section{A. Adaptive Negotiation Model Based Automation Design}

This automation design is based on the adaptive negotiation model introduced in [4]. Its objective is to model a humanmachine negotiation over a set of decision options $\mathcal{O}$ by exchanging offers $o_{i}^{t}$ among participating agents $i \in\{A, H\}$ (Automation \& Human) at times $t$. The core component is a customized basic negotiation model (cf. [3], [20]): Agents interact according to the negotiation protocol, evaluate offers by means of an individual utility function and accept or generate offers via acceptance and bidding strategies. This basic model is enhanced by an identification module ${ }^{1}$ and an adaptation concept. Although humans may also identify the negotiation behavior of the cooperation partner and adapt towards it, we only model these aspects for the automation in this paper. An overview of the overall adaptive negotiation model and the interaction of its component is provided in Fig. 2. In the following, we briefly introduce the different components of the adaptive negotiation model.

1) Basic Negotiation Model: In our previous work, we demonstrated the suitability of the basic negotiation model to model human negotiation behavior in only-concession cooperative decision making scenarios [6]. However, this required some prior customization of the basic negotiation model for its application in the context of human-machine cooperation. As a result, it consists of the following parts considering both agent $i \in\{A, H\}$ :

- A normalized utility function $U_{i}$ for evaluating offers $O$ i. e. $U_{i}(o) \in[0,1] \subset \mathbb{R}$.

- An acceptance strategy $S_{i}$ that accepts an offer $o_{j}^{\kappa}$ of offer history $\mathcal{O}_{j}^{\mathrm{H}}$ established by the cooperation partner $j \in$ $\{A, H\}, j \neq i$, if this offer is also present in the own offer history $\mathcal{O}_{i}^{\mathrm{H}}$ of agent $i$, i. e. agent $i$ has already proposed this offer before.

- A bidding strategy for determining a (counter) offer $o_{i}^{k+1}$ that is set to be a time-based concession strategy $C_{i}$ due to the fact that there is usually some sort of deadline $T$

\footnotetext{
${ }^{1}$ Also called opponent model in the context of negotiation theory. However, in this paper we consider the cooperation partner not as an opponent although objectives may be different.
}

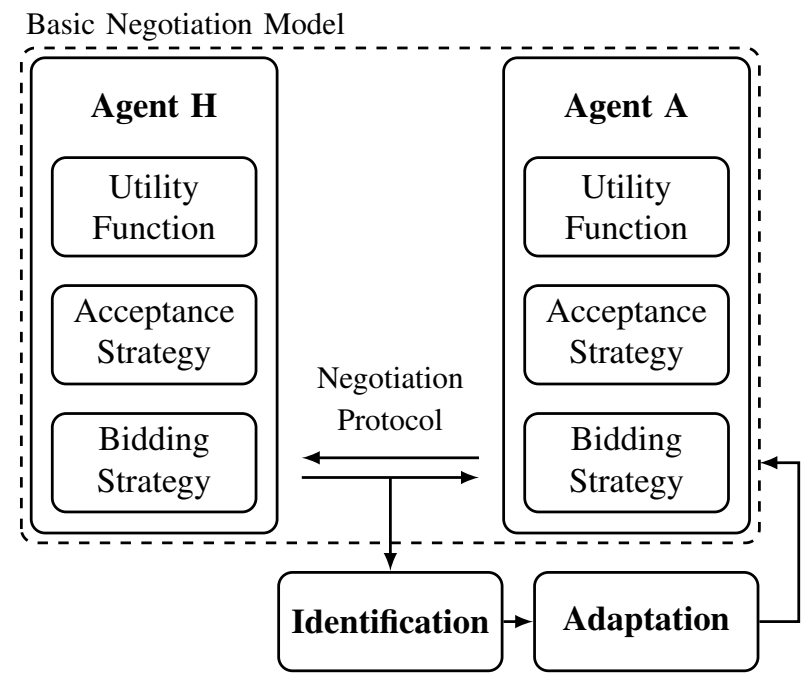

Fig. 2. Overview of the adaptive negotiation model [4].

present in human-machine cooperative decision making scenarios. The concession is modeled via a target utility $U_{\mathrm{t}, i}(t)$ that is decreasing over time:

$$
U_{\mathrm{t}, i}(t)=\max _{o \in \mathcal{O}} U_{i}(o) \cdot\left(1-\left(\frac{t}{T}\right)^{1 / \varepsilon_{i}}\right) .
$$

The parameter $\varepsilon_{i}$ is called concession rate. The agent tries to track this target utility with the own offers' utility values. Hence, new offers $o_{i}^{k}$ at time $t_{k}$ are proposed and added to the offer history set $\mathcal{O}_{i}^{\mathrm{H}}$ if $U_{i}\left(o_{i}^{k}\right) \geq U_{\mathrm{t}, i}\left(t_{k}\right)$ and if the offer has not yet been proposed, i.e. it is not yet part of the history set $\mathcal{O}_{i}^{\mathrm{H}}$.

- An asynchronous negotiation protocol [3], allowing the agents to communicate, i.e. exchanging offers, on an event basis which suits human communication behavior.

2) Identification: In order to influence the outcome of the negotiation and enhance the time-based concession of the basic negotiation model, agents may use the information from their cooperation partner's offers to identify his utility function and negotiation behavior in terms of the bidding strategy, and apply this knowledge within their own bidding strategy. Hence, the automated agent applies a Bayesian learning approach [21] to identify the human negotiation behavior.

3) Generalized Adaptation Approach: With the information regarding the human's negotiation behavior, the automated agent is able to adapt its bidding strategy. In contrast to the implicit use of this information within the bidding strategy in state-of-the-art approaches (cf. [22], [23]), we introduced a generalized adaptation principle [4] which relies on the prediction of the negotiation outcome in terms of utility and the effort to achieve this outcome.

For further information on the adaptive negotiation model and respective automation design refer to [4].

\section{B. The n-Stage War of Attrition Automation Design}

The basis of this automation design is the $n$-stage war of attrition [7]. 
1) The Game Model: The model is a continuous-time, incomplete information game with two rational players $\{i, j\}=$ $\mathcal{P}$. The players face a set of decision options $\mathcal{O}$ and each player individually assigns unique values $v_{o}$ for the game being terminated with an agreement on the different decision options $o$ and a universal value of $-\infty$ for terminating the game in disagreement. Due to the fact that this valuation is private information of the individual player, players will start to offer the decision options corresponding to their highest values. In case both initial decision option offers are identical, an agreement is found and the game terminates instantaneously. However, if no instant agreement is found, players are confronted with a time-dependent disagreement cost function $c(t): \mathbb{R}^{+} \rightarrow \mathbb{R}^{+}$ that is strictly increasing $\left(\frac{\mathrm{d}}{\mathrm{d} t} c(t)=c^{\prime}(t)>0 \forall t \in \mathbb{R}^{+}\right)$and identical for both players. It reduces the players' agreement valuation of chosen decision options over time. This setting forces the rational, and therefore payoff-maximizing players to concede to less valuable decision options at certain time thresholds $\tau$. Consequently, both players build up an offer history set $\mathcal{O}^{H}$ of decision options throughout the game. An agreement is found as soon as $\mathcal{O}_{i}^{H} \cap \mathcal{O}_{j}^{H}=o_{f} \neq \emptyset$ which also terminates the game.

To facilitate the following description of threshold determination, the thresholds are used to split the game in stages. The theoretical maximum number of stages is equivalent to the number of decision options $|\mathcal{O}|=n$. Each stage $k$ is associated with a valuation difference $d^{k}$ for each player. $d_{i}^{k}$ are defined as the difference between the valuation of the decision option player $i$ favors at the beginning of stage $k$ and the valuation of the decision option player $i$ potentially concedes to at the end of stage $k$. In contrast to the valuations itself, players' density distribution of all valuation differences $f_{d}$ are known by the other player.

2) The Solution Strategy: To find the solution strategy of the game, i.e. the thresholds $\tau_{i}^{k}$, for each player, it is assumed that players treat the current stage as if it was the last stage of the game. This is motivated by the incomplete information stetting and the typically bounded rationality of humans [24]. Furthermore, in this setting players will maximize their expected payoff. Consequently, the threshold $\tau_{i}^{k}$ of player $i$ depends on his own valuation difference $d_{i}^{k}$ of the current stage $k$, the density distribution of valuation differences $f_{d}$ of the other player $j$ and the general cost function $c(t)$.

For further information on the threshold calculations and the resulting equilibrium refer to [7].

3) The Automation Design: The automation offers the decision options according to the description in Section II-B1 and determines the required thresholds. Obviously, a metric valuation measure for the decision options is required to determine $d_{A}$. Furthermore, due to the usual lack of information on the density distribution $f_{d}$ of valuation differences of the human player, a uniform distribution on an appropriate range of valuation differences is initially utilized. With repetitions of the game, this density distribution can be updated. One possible approach is introduced in our previous paper [7].

\section{State-of-the-art Leader-Follower Automation Design for Comparison}

In the context of human-machine interaction on decision level, state-of-the-art automation designs eventually follow a leader-follower paradigm (cf. Section I-A). In essence, the leader has full control and authority whereas the follower may present suggestions and assist the leader but ultimately has to follow the leader's commands.

As a consequence, there are two possible automation designs along with the leader-follower paradigm: One design puts the human in the lead and the automation as the follower, i. e. the automation may propose its choices but ultimately accepts the human decision. The other design sets the automation as the leader and the human as the follower.

\section{Comparing Automation Designs}

In the next section, we experimentally compare these two leader-follower-based automation designs with the two automation designs based on the cooperative decision making models introduced in Sections II-A and II-B. In contrast to the leader-follower-based automation designs, these new automation designs based on cooperative decision making models display not only suggestions but also exhibit concession behavior in conflict situations. The new automation designs differ in their extent of concession: the negotiation-theorybased automation design will give in as a last resort whereas the game-theory-based automation design ultimately insists and realizes its decision in case no agreement is reached. Hence, these two automation designs close the gap between the two extremes of the leader-follower approaches in terms of authority.

To easily differentiate the four automation designs in the following, we utilize the following abbreviations: automation design based on Game Theory (GT) and Negotiation Theory (NT); automation designs based on the leader-follower approach with the Leader being the Human (LH) or the Leader being the Automation (LA).

\section{EXPERIMENTAL DESIGN}

In general, experiments that investigate cooperative decision making have to provide decision scenarios with a set of decision options and a possibility for the cooperation partners to communicate. Additionally, the cooperation partners have to be able to evaluate the decision options and (sufficiently often) find themselves in a situation with conflicting preferences (either due to different evaluation criteria or differences in the information they obtained) requiring an interaction process to reach an agreement. Furthermore, for reasons of practicality, a pressure for reaching an agreement should be present.

Taking the above into account, this section provides one solution to our research question $\mathrm{R} 2$ that is a suitable experimental design to investigate the benefits of humanmachine cooperation on decision level by comparing our automation designs based on the cooperative decision making models, i. e. GT and NT, with state-of-the-art leader-follower approaches, i.e. LH and LA, cf. Section II-D. From our perspective a suitable design of such an experiment has to 
meet the following requirements: a) provide a controlled but realistic and dynamic environment with b) multiple decision scenarios that c) are intuitively comprehensible by participants and d) allow for differences in decision options preferences between human and automation, e) enable a human-machine cooperation on decision level in which no partner is always able to outperform the other, f) provide objective and subjective measures to evaluate cooperative performance, g) avoid confounding variables esp. in human-machine communication, and $h$ ) ensure a repeatability of experimental runs considering the four investigated automation designs and i) avoid learning effects during the course of the experiment.

To satisfy these requirements, we chose a futuristic yet reasonable highly automated driving scenario: We a) utilized a driving simulator (cf. Fig. 3) to realistically recreate a drive in a highly automated vehicle through a so called Manhattan grid with b) multiple intersections, each representing a cooperative decision scenario in which a direction (left, right, straight ahead) has to be chosen. This choice is c) influenced by potential traffic delays associated with specific vehicles at different directions and the objective to minimizing travel time to reach a defined destination displayed on a map. Additionally, participants are made aware of their cooperative decision making performance with the automation by means of an objective performance measure based on the minimal travel time: after each decision making the deviation in travel time between the locally chosen direction and the optimal global choice is displayed as well as the overall time deviation between the optimal and chosen path. In order to d) create decision conflicts, the human participant is only aware of the traffic at the next intersection (local information) whereas the automation has information on all traffic (global information). However, the information of the automation is e) partially false (e.g. due to inaccurate perception of the dynamic traffic development at the next intersection) such that the cooperation of human and automation, i.e. the intervention of the human, potentially yields benefits which are observable via the displayed objective performance measure. The cooperative performance is f) objectively evaluated by means of the travel time and subjectively via a questionnaire assessing the satisfaction with the cooperation, the intuition of interaction, and the reliability and transparency of the partner's behavior. To avoid confounding factors, g) the human and the automation solely communicate via a cooperative maneuver decision making interface (CMDI) consisting of a head-up display and a touchscreen. The interface provides the discrete decision options of the next intersection and the remaining time until a mutual decision has to be reached before entering this intersection. When reaching the intersection the mutually chosen decision option represents the driving maneuver the highly automated vehicle then executes until the next intersection. For each drive through the Manhattan grid, i. e. each experimental run, associated with the application of a different decision making automation design, participants are h) confronted with the same Manhattan grid setup but will be unaware as the displayed map is rotated by a defined multiple of $90^{\circ}$. Furthermore, the sequence of experimental runs within the experiment is i) randomized for each participant.

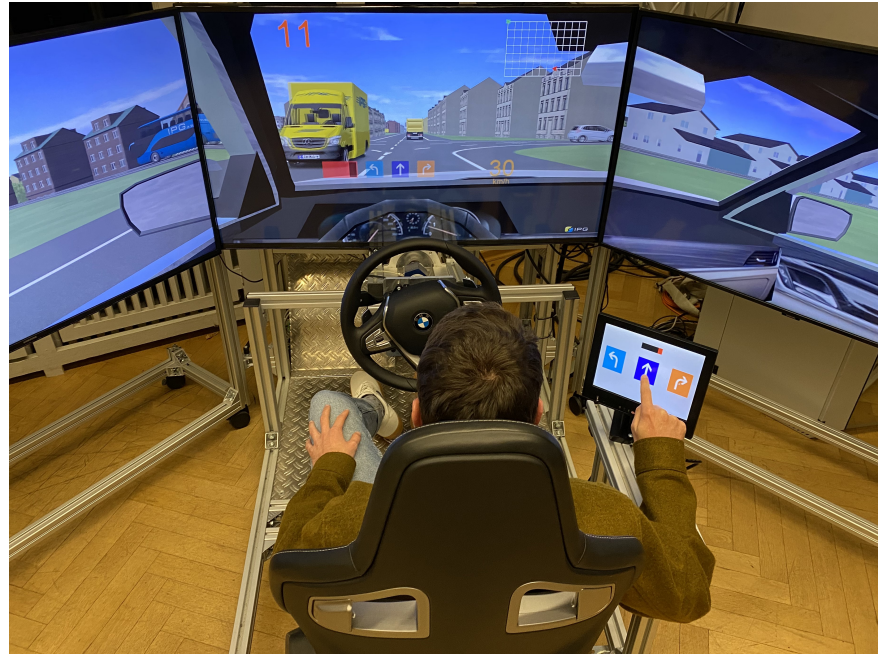

Fig. 3. Front view of the driving simulator for highly automated driving equipped with three vehicle visualization screens (top), steering wheel and pedals (middle, unused in this experiment), a driver's seat (bottom) and a touchscreen as a maneuver decision interface (mid-right).

\section{A. Setup}

The experiment's setup is based on a simulator for highly automated driving developed by the Institute of Control Systems (IRS) at KIT with a human-machine interface on driving maneuver level for cooperative decision making, see Fig. 3. Its core is a XPACK4 real-time system from IPG Automotive $G m b H$ and their vehicle simulation software CarMaker ${ }^{\circledR} 8$. This setup is utilized to simulate the driving behavior of a car and its environment including traffic. For this experiment the hardware setup is enhanced by three visualization screens displaying the simulated vehicle, its surroundings and a headup display as the visual part of the CMDI. Furthermore, a touchscreen is integrated on the right hand side of the driver's seat as the input device of the CMDI. Additionally, a sound system provides driving sounds and other userdesigned sounds, i. e. warning signals. The simulation software is enhanced by a customized vehicle control module for highly automated driving and for cooperative decision making based on the four decision making automation designs.

The visual part of the CMDI is displayed on the middle screen as a head-up display (cf. Fig. 4) and consists of the following components:

- The available maneuvers, i. e. directions, at the next intersection (cf. Section III-B), indicated by icons displaying respective arrows. The icons' background colors indicate the maneuvers' current status: gray indicates the nonavailability of maneuver options, light blue indicates their availability; orange signals the automation's choice of maneuver (and history), dark blue signals the maneuver choice (history) of the driver; and green informs about an agreement on the corresponding maneuver.

- A countdown of $3 \mathrm{~s}$, motivated by previous experiences [6] and displayed by means of a series of yellow triangles with respective numbers, before a cooperative decision making is enabled.

- A bar graph with a red background and a black rectangle, 


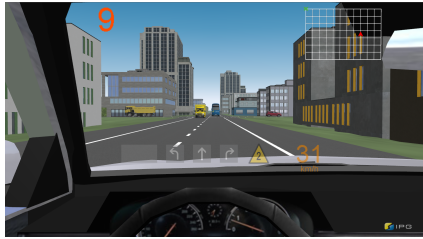

(a) Countdown phase prior to a cooperative decision making phase with disabled bar graph and decision options in gray color.

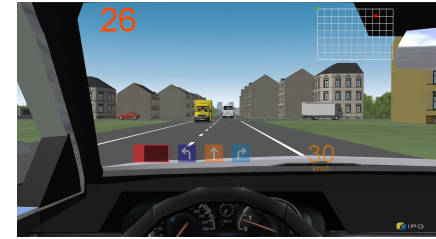

(b) Situation in a cooperative decision making phase with one maneuver choice of a participant (straight ahead, orange color) and the automation (left, dark blue color), the not chosen but available maneuver (right, light blue) and the bar graph (red \& black).

Fig. 4. Exemplary screenshots of the simulator's middle screen including the head-up display containing the display of a cooperative performance measure (top left), the available directions i.e. maneuvers (center), the countdown display (right of center), a bar graph indicating the remaining time until the deadline (left of center) and the current vehicle speed (far right of center).

the size of which corresponds to the remaining time until the predefined deadline in a period of cooperative decision making is reached.

Additionally and only for experimental design reasons, the objective measure of cooperative performance, associated with the travel time and explained further in Section III-E, is displayed outside of the CMDI on the middle screen's top left corner, as well as a map of the overall Manhattan grid (cf. Section III-B) in the top right corner (cf. Fig. 3 and 4). The map is showing the current position of the vehicle and the destination but no other traffic.

\section{B. Decision Scenarios}

In general, decision scenarios comprise a set of decision options that agents are able to evaluate individually. If agents have to cooperatively decide on one decision option the following types of decision scenarios are possible:

- Conflict: In this scenario type, both agents have strong opposing preferences on the choice of a specific decision option. Hence, agents are forced into a cooperative decision making process.

- Persuasion: In these scenarios, one agent is almost indifferent towards the decision options while the other agent has a strong preference and therefore is expected to try to persuade the other agent.

- Trivial: Both agents prefer the same decision option and no process to reach an agreement is required.

To provide a natural flow of comparable, realistic and intuitive decision scenarios, we simulate a goal-directed drive through the Manhattan grid leading to a series of four-way intersections. Each intersection represents a decision scenario with different decision options, i. e. driving maneuvers: drive left, right, straight ahead (cf. Fig. 5). A potential local traffic delay based on different types of vehicles (i.e. car, van, bus, truck) causing different but known delays is associated with each maneuver option. These delays can be contrasted to the time that it takes to travel from one intersection to the next one without any traffic delays which is $14.5 \mathrm{~s}$ : car $+3.6 \mathrm{~s}(+25 \%)$, van $+7.3 \mathrm{~s}(+50 \%)$, bus $+14.5 \mathrm{~s}(+100 \%)$, truck

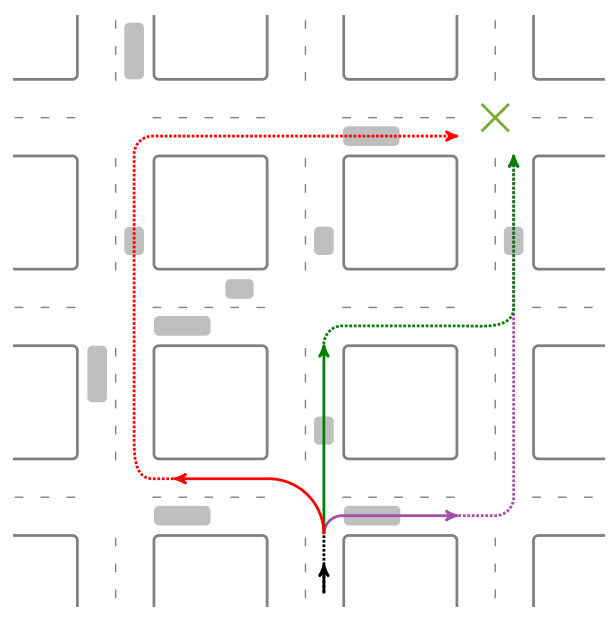

Fig. 5. Exemplary part of the Manhattan grid indicating traffic delays by gray rectangles (lengths represent the delay duration) and presenting the three decision options, i. e. maneuver options, for one decision scenario at the corresponding intersection by solid colored arrows. Respective optimal future paths to the destination $\times$ are depicted with dotted lines.

$+29 \mathrm{~s}(+200 \%)$. In the following, a delay step or travel time step is defined for reasons of simplicity and readability as $3.6 \mathrm{~s}$, i. e. the delay of a car.

When driving through the Manhattan grid, human participants and the automation are aware of their current position and destination by means of the displayed map. Participants are also aware of the local traffic when approaching the intersection. Hence, they are able to assess the associated local delays. The automation has global information about the general traffic delays at all subsequent intersections (motivated by state-of-the-art real-time traffic information distribution and future car-to-x technology), yet it may have false information about the local traffic delays at the next intersection (simulating the environment perception of the automation that requires some time for local information updating). The automation is therefore able to evaluate the global and potentially inaccurate local delays for each decision option at the current intersection with respect to the destination.

This setting emphasizes the strength of both cooperating partners: The automated vehicle is well informed regarding the traffic along the upcoming route but can be tainted by potentially misinterpreted delays due to changing local traffic. The human is not able to anticipate future traffic but perceives local traffic information correctly.

Consequently, local delays as well as misinformation are purposefully applied in the design of the Manhattan grid to create different maneuver preferences for human participants and the automation at each intersection, yielding the different types of decision scenarios:

- Conflict: Both agents have strong opposing preferences on the choice of a specific driving maneuver, i. e. there are at least three delay steps between first and second maneuver preference for each agent and agents disagree on first and second preferences.

- Persuasion: One agent is almost indifferent between first and second maneuver preferences, because there is only one delay step in between, while the other agent has a 
strong preference (minimum 3 delay steps) and therefore is expected to try to persuade the other agent.

- Trivial: Both agents evaluate the same maneuver option as the best. This type of scenario is applied to show the human the potential immediate agreement and that there is not always a conflict.

The overall size of the Manhattan Grid is $12 \times 8$ intersections consisting of 29 conflict scenarios, 33 persuasion scenarios and 30 trivial scenarios, disregarding the grid's corners. The start position of the automated vehicle and the destination are placed on opposite corners of the grid. The globally optimal path to reach the destination without misinformation consists of 6 conflict scenarios, 8 persuasion scenarios and 3 trivial scenarios. On this optimal path, traffic delays accumulate to 29 steps which is used as a baseline to compare the performance of the four different automation design to.

Each decision scenario starts with a countdown of $3 \mathrm{~s}$. Within this time period the human agent is able to perceive the local traffic information regarding the upcoming intersection and the vehicle's position on the map. After the countdown, the actual phase of cooperative decision making is starting while the human agent is asked to communicate his most preferred option first. Afterwards, the automation will present its most preferred option. Depending on how strong or weak the preferences are, the automation designs based on the cooperative decision making models as well as the participants are expected to give in after some time (and potentially iterations) and agree with the cooperation partner on the respective maneuver choice. In case of the LA automation design or stubborn human behavior no agreement may be reached. Then the final decision is set according to the current automation design as explained in Section II-D. Hence, the phase of cooperative decision making ends either by an agreement on one maneuver option or by reaching the predefined deadline, i. e. the vehicle is entering the intersection, after $9 \mathrm{~s}$. This time is motivated by the assumption of at most three choices with $3 \mathrm{~s}$ each, as applied in [6]. The remaining time until reaching the deadline and entering the intersection is displayed by means of the bar graph for more clarity. After the deadline is reached, the resulting maneuver option as well as the current, updated measure of cooperative performance and its potential increase are displayed. The increase describes the potentially added travel time steps of the resulting option with respect to the optimal path from the current intersection to the destination. Furthermore, the participant actually experiences the potential local traffic delay because the automated vehicle is slowed down depending on the traffic associated with the conducted maneuver. This traffic disappears before the next decision scenario starts.

With respect to the model-based automation design of Section II, the local and global delay information of maneuver options is translated directly to their utilities (NT) and valuation differences (GT) of the cooperative decision making models. To account for differences regarding the maximum and minimum delays of available maneuver options at different intersections, data of each decision scenario were normalized.

\section{Procedure}

In the following, we introduce the experiment's procedure. The overall practical accomplishment of the experiment took between 45 and $60 \mathrm{~min}$.

1) Introduction and Preparations: Participants first read the guidelines on how to conduct the experiment. They were informed about the setup of the decision scenarios, i.e. explaining the Manhattan grid with intersections consisting of (usually) three decision options, the delays caused by the different types of vehicles at the intersections and the time to deadline. In addition, they were informed that the automation places maneuver option offers based on information about additional delays at subsequent intersections and potentially false information about local delays. The objective for the participants was to reach a marked destination in the shortest possible time by iteratively and cooperatively decide on a travel route. In each of the following experimental runs, they were unaware of the type of automation design i.e. the exact maneuver-choosing behavior of the automation. Finally, the participants were asked to fill out the part of the customdesigned questionnaire regarding their general information and the familiarization procedure started.

2) Familiarization Procedure: To introduce the general procedure of different decision scenarios and the handling of the decision interface, the participants were facing a shortened Manhattan grid $(6 \times 8)$ which consisted of random combinations of decision scenarios and automation designs. The results of this part are not included in the evaluation.

3) First to Fourth Experimental Run: For each of the four automation designs the participants were passing one experimental run. The order of experimental runs were counterbalanced over participants to equate potential learning effects. Each experimental run was evaluated by the participants via a specific section of the custom-designed questionnaire. This scheme was applied to strengthen their sensitization and contemplation regarding the different automation designs.

4) Postprocessing: After completing the fourth experimental run, the participants were asked to fill out the last part of the given questionnaire which enabled an evaluation of the four experimental runs in relation to each other.

\section{Participants}

33 participants (27 male and 6 female) took part in the experiment. The average age was 29 years with an age range of 22 to 57 years. All participants possessed a valid driving license and $30.3 \%$ did have some general experience regarding driving simulators.

\section{E. Measures}

The relevant measures for this paper are an objective cooperative performance measure and subjective assessment by means of a questionnaire to evaluate the four experimental runs: We compare the two automation designs based on the cooperative decision making models with the two automation designs following the leader-follower approach. Furthermore, we also analyse the relation of all four automation designs to each other. 
The objective cooperative performance regarding the human-machine cooperative decision making is measured by the additional travel time steps when comparing the required travel time at the end of each experimental run to the optimal route's travel time. Hence, the smaller the additional travel time steps, the higher the performance of the human-machine cooperation.

To assess the participants' subjective perception of the human-machine cooperation, a questionnaire with a five-point Likert Scale [25] with the following items was used.

Q1: How do you assess the overall cooperation between you and your partner?

Answer range from not satisfying (1) to satisfying (5).

Q2: How do you assess your partner's cooperation behavior? Answer range from not reliable (1) to reliable (5).

Q3: How do you assess the interaction between you and your partner?

Answer range from not intuitive (1) to intuitive (5).

Q4: Was the behavior of your partner in cooperative decision making transparent?

Answer range from not transparent (1) to transparent (5).

By means of the non-parametric Kruskal-Wallis test [26] the statistical analysis is conducted as we compare up to four sample sets and lack information regarding their distributions. The test's null hypothesis (all sample sets origin from the same original distribution) is accepted if $\mathcal{H} \leq \chi_{\text {crit }}^{2}$ holds. In case of the pooled comparison of the two automation designs based on the state-of-the-art leader-follower approach (LH \& LA) with the two newly-introduced automation designs based on the cooperative decision making models (GT \& NT) $\chi_{c r i t}^{2}=\chi_{d f=1, \alpha=0.05}^{2}=3.842$ follows. When comparing the individual results of the four automation designs, there are three degrees of freedom $(d f=3)$. Hence, with a significance level of $\alpha=0.05, \chi_{c r i t}^{2}=\chi_{d f=3, \alpha=0.05}^{2}=7.815$ follows.

Based on these measures, the following section provides the results of the conducted experiment.

\section{Results}

Fig. 6 shows the objective cooperative performance by means of compact boxplots based on the additional travel time steps for each automation design. It reveals that experimental runs with the automation designs based on cooperative decision making models yielded less additional time steps than the leader-follower-based automation designs. Furthermore, comparing the pooled leader-follower-based automation designs LA \& LH with the pooled automation designs GT \& NT, the null hypothesis of the Kruskal-Wallis test is declined with $\mathcal{H}=72.123$. Considering the sample set for the four automation designs individually, the null hypothesis is declined with $\mathcal{H}=64.823$. Hence, the objective cooperative performance measure was significantly better for the automation designs based on cooperative decision making models than for the leader-follower automation designs.

Fig. 7 shows the participants' subjective perceptions based on the corresponding questions Q1-Q4 of the questionnaire. Comparing the pooled automation designs LA \& LH with the pooled automation designs GT \& NT, the null hypothesis of

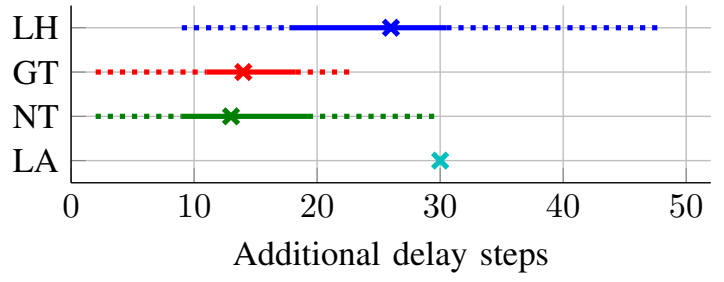

Fig. 6. Compact boxplots of additional travel time steps for each automation design. Median $\times$, lower/upper quartile - , lower/upper adjacent $\cdots$.

TABLE I

RESULTS OF THE T-TEST EVALUATING OBJECTIVE PERFORMANCE MEASURE AND ANSWERS TO Q1-Q4: P-VALUES OF PAIR-WISE COMPARISON.

\begin{tabular}{lcccccc}
\hline & LAirs & LA & LA & NT & NT & GT \\
measures & NT & GT & LH & GT & LH & LH \\
\hline obj. coop. perf. & 0 & 0 & 0.042 & 1 & 0 & 0 \\
\hline Q1: satisfaction & 0 & 0 & 1 & 1 & 0 & 0 \\
\hline Q2: trust & 0 & 0 & 1 & 1 & 0 & 0 \\
\hline Q3: intuition & 0.004 & 0 & 1 & 1 & 0.022 & 0.003 \\
\hline Q4: transparency & 1 & 1 & 0.304 & 1 & 0.016 & 0.026 \\
\hline
\end{tabular}

the Kruskal-Wallis test is declined regarding the satisfaction with the human-machine cooperation $(\mathcal{H}=83.776)$, the trust in automation's decision making behavior $(\mathcal{H}=52.51)$, the intuition of the interaction $(\mathcal{H}=24.192)$ and the transparency of the interaction $(\mathcal{H}=7.563)$. In view of the individual sample sets of the four automation designs, the null hypothesis of the Kruskal-Wallis test is also declined regarding the satisfaction with the human-machine cooperation $(\mathcal{H}=84.845)$, the trust in automation's decision making behavior $(\mathcal{H}=52.682)$, the intuition of the interaction $(\mathcal{H}=24.85)$ and the transparency of the interaction $(\mathcal{H}=11.406)$. To sum up, the evaluation of subjective perception regarding the different automation designs revealed that the automation designs based on cooperative decision models lead to a significantly more satisfying, trustworthy and intuitive interaction in comparison to the stateof-the-art leader-follower approaches. However, the opposite holds for the transparency of the interaction.

In summary, the hypotheses stated in Section I-C were all confirmed.

For a deeper understanding, we also provide some posttest results for each measure comparing the sample sets of each automation design individually by means of a ttest. All resulting p-values are given in Table I. Considering the objective cooperation performance measure, all sample sets differ significantly except for the comparison of NT \& GT. Regarding the participants' satisfaction with the humanmachine cooperation, the trust in the automation's decision making behavior and the intuition of the interaction between human and automation, the sample sets of both NT and GT are significantly different compared to LH and LA. Considering the transparency of the interaction between human and automation, there are significant differences comparing the sample sets of GT and NT with LH and no significant difference in comparison with LA. 


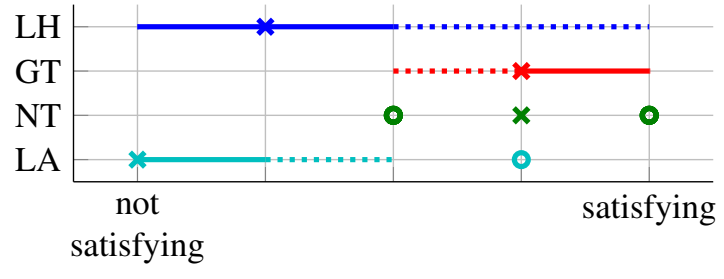

(a) Q1: satisfaction regarding the human-machine cooperation

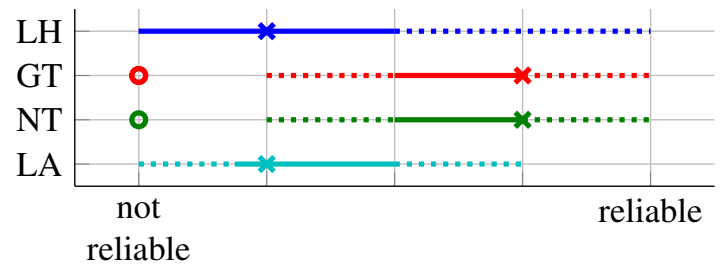

(b) Q2: trust in automation's decision making behavior

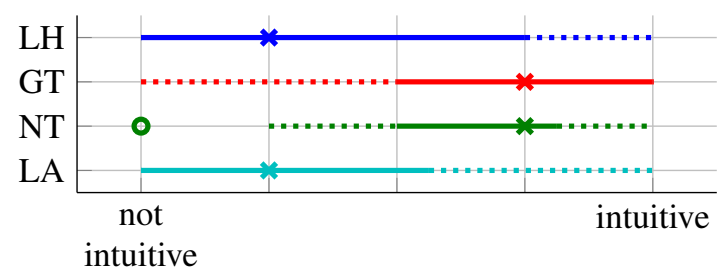

(c) Q3: intuition of the interaction

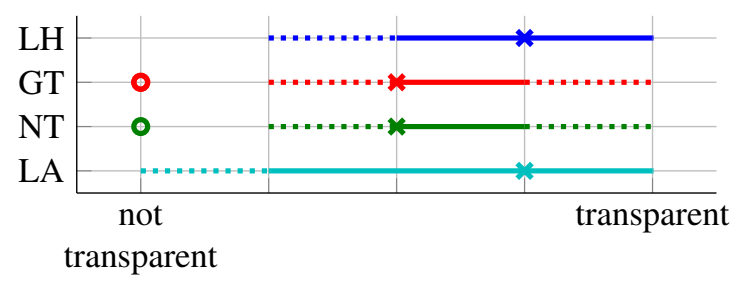

(d) Q4: transparency of the interaction

Fig. 7. Compact boxplots regarding the subjective perceptions to Q1-4. Median $\times$, lower/upper quartile - , lower/upper adjacent $\cdots$, outlier $\circ$.

Furthermore, we found that the objective cooperation performance measure strongly correlated to participants' subjective assessment of the satisfaction with the human-machine cooperation $(M=-0.8113, S D=0.2195)$. In other words, participants were more satisfied with the human-machine cooperation if the cooperation led to smaller travel times (a better performance), and vice-versa.

The above gained insights were also supported by collected statements of participants noticing a "will to compromise" and "good proposals" of the automation designs based in the cooperative decision making models. The interaction with them was perceived as "pleasant" and "trustworthy". The interaction with leader-follower approaches was criticized as "frustrating" and "strenuous". Participants perceived the automation design with the automation in lead as "too dominant" and "unresponsive to suggestions". When participants were in lead the automation was criticized for "taking no responsibility".

\section{DisCusSION}

The significantly improved objective cooperative performance for the automation designs based on cooperative deci- sion making models compared to the leader-follower automation designs demonstrates a) that an emancipated design of the human-machine cooperation on decision level is beneficial for the overall cooperative system's performance and b) that a model-based approach is suitable to design the corresponding automation.

The observed significantly more satisfying and intuitive interaction with the automation designs based on cooperative decision models is in our view a result of the significantly increased trust regarding the automation's decision making behavior. In other words, participants recognized the increased cooperative, i. e. concessive, behavior of the introduced cooperative decision model automation designs as more trustworthy and intuitive which also increases participants' acceptance of the automation.

A closer look at the reduced transparency of interaction for the automation designs based on cooperative decision making models reveals two insights: a) Designing fully automated systems is not necessarily the solution in terms of transparency for humans as interaction with LA was not assessed significantly more transparent than GT \& NT. b) Humans prefer complete transparency about the final decision as interaction with LH is assessed significantly more transparent compared to all other automation designs which was expected. Hence, in terms of transparency, assistive decision support systems have an advantage.

Putting together all these insights, the trade-off in designing cooperative systems becomes apparent, i.e. balancing the aspects of cooperative performance, human acceptance, trust in the automation, intuition and transparency of interaction. According to the experiment's results and depending on the context, approaches with focus on cooperative decision making or humans in lead are preferable in contrast to approaches with the automation in lead.

\section{CONCLUSION}

The experiment reported in this paper yields results which demonstrate that the proposed automation designs for cooperative decision making based on negotiation theory and game theory add value for human-machine cooperation on decision level in the examined scope of highly automated vehicles: the objective cooperative performance was increased compared to automation designs based on conventional leader-follower approaches. Additionally, participants' subjective assessment in terms of satisfaction and trust in the cooperation as well as intuition of interaction was better for cooperative decisions models while the transparency of interaction slightly decreased as expected.

Hence, the experiment yields new insight for the field of human-machine cooperation by providing first evidence of humans' preference for a truly equal cooperative interaction on a decision level.

\section{REFERENCES}

[1] D. A. Abbink, T. Carlson, M. Mulder, J. C. F. de Winter, F. Aminravan, T. L. Gibo, and E. R. Boer, "A topology of shared control systemsfinding common ground in diversity," IEEE Transactions on HumanMachine Systems, vol. 48, no. 5, pp. 509-525, 2018. 
[2] M. Flad, "Differential-game-based driver assistance system for fueloptimal driving," in Frontiers of Dynamic Games, ser. Static \& Dynamic Game Theory: Foundations \& Applications, L. A. Petrosyan, V. V. Mazalov, and N. A. Zenkevich, Eds. Cham: Springer International Publishing, 2019, pp. 13-36.

[3] S. Rothfuss, R. Schmidt, M. Flad, and S. Hohmann, "A concept for human-machine negotiation in advanced driving assistance systems," in 2019 IEEE International Conference on Systems, Man and Cybernetics (SMC). IEEE, 2019, pp. 3116-3123.

[4] S. Rothfuss, C. Ayllon, M. Flad, and S. Hohmann, "Adaptive negotiation model for human-machine interaction on decision level," in 2020 IFAC World Congress. IFAC, 2020.

[5] S. Rothfuss, J. Steinkamp, M. Flad, and S. Hohmann, "Cooperative decision making in cooperative control systems by means of game theory," in Frontiers of Dynamic Games. Game Theory and Management, St. Petersburg, 2019. Birkhauser, 2020.

[6] S. Rothfuß, M. Wörner, J. Inga, and S. Hohmann, "A study on humanmachine cooperation on decision level," in 2020 IEEE International Conference on Systems, Man, and Cybernetics (SMC). IEEE, 2020, pp. 2291-2298.

[7] S. Rothfuß, T. S. Tanaka, J. Inga, and S. Hohmann, "The n-stage war of attrition and its inverse game towards its application in humanmachine cooperative decision making," in 2020 59th IEEE Conference on Decision and Control (CDC). IEEE, 2020, pp. 5386-5393.

[8] F. Flemisch, D. Abbink, M. Itoh, M.-P. Pacaux-Lemoine, and G. Weßel, "Shared control is the sharp end of cooperation: Towards a common framework of joint action, shared control and human machine cooperation," IFAC-PapersOnLine, vol. 49, no. 19, pp. 72-77, 2016.

[9] E. Donges, "A conceptual framework for active safety in road traffic," Vehicle System Dynamics, vol. 32, no. 2-3, pp. 113-128, 1999.

[10] R. Parasuraman, T. Sheridan, and C. Wickens, "A model for types and levels of human interaction with automation. ieee trans. syst. man cybern. part a syst. hum. 30(3), 286-297," IEEE transactions on systems, man, and cybernetics. Part A, Systems and humans : a publication of the IEEE Systems, Man, and Cybernetics Society, vol. 30, pp. 286-297, 2000.

[11] J. Rasmussen, "Skills, rules, and knowledge; signals, signs, and symbols, and other distinctions in human performance models," IEEE Transactions on Systems, Man, and Cybernetics, vol. SMC-13, no. 3, pp. 257266, 1983.

[12] M. Della Penna, M. M. van Paassen, D. A. Abbink, M. Mulder, and M. Mulder, "Reducing steering wheel stiffness is beneficial in supporting evasive maneuvers," in 2010 IEEE International Conference on Systems, Man and Cybernetics. IEEE, 2010, pp. 1628-1635.

[13] A. Mortl, M. Lawitzky, A. Kucukyilmaz, M. Sezgin, C. Basdogan, and S. Hirche, "The role of roles: Physical cooperation between humans and robots," The International Journal of Robotics Research, vol. 31, no. 13, pp. 1656-1674, 2012.

[14] X. Na and D. J. Cole, "Game-theoretic modeling of the steering interaction between a human driver and a vehicle collision avoidance controller," IEEE Transactions on Human-Machine Systems, vol. 45, no. 1 , pp. 25-38, 2015.

[15] J. R. Medina, T. Lorenz, and S. Hirche, "Synthesizing anticipatory haptic assistance considering human behavior uncertainty," IEEE Transactions on Robotics, vol. 31, no. 1, pp. 180-190, 2015.

[16] S. O. Oguz, A. Kucukyilmaz, T. M. Sezgin, and C. Basdogan, "Supporting negotiation behavior with haptics-enabled human-computer interfaces," IEEE transactions on haptics, vol. 5, no. 3, pp. 274-284, 2012.

[17] R. Groten, D. Feth, R. L. Klatzky, and A. Peer, "The role of haptic feedback for the integration of intentions in shared task execution," IEEE transactions on haptics, vol. 6, no. 1, pp. 94-105, 2013.

[18] F. Flemisch, H. Winner, R. Bruder, and K. Bengler, "Cooperative guidance, control and automation," in Handbook of Driver Assistance Systems: Basic Information, Components and Systems for Active Safety and Comfort, H. Winner, S. Hakuli, F. Lotz, and C. Singer, Eds. Cham: Springer International Publishing, 2016, pp. 1471-1481.

[19] M. Walch, M. Woide, K. Mühl, M. Baumann, and M. Weber, "Cooperative overtaking," in Proceedings of the 11th International Conference on Automotive User Interfaces and Interactive Vehicular Applications AutomotiveUI '19. New York, New York, USA: ACM Press, 2019, pp. 144-155.

[20] T. Baarslag, E. H. Gerding, R. Aydogan, and M. C. Schraefel, "Optimal negotiation decision functions in time-sensitive domains," in 2015 IEEE/WIC/ACM International Conference on Web Intelligence and Intelligent Agent Technology (WI-IAT). IEEE, 2015, pp. 190-197.

[21] K. Hindriks and D. Tykhonov, "Opponent modelling in automated multiissue negotiation using bayesian learning," in Proceedings of the 7th
International Joint Conference on Autonomous Agents and Multiagent Systems - Volume 1, ser. AAMAS '08. Richland, SC: International Foundation for Autonomous Agents and Multiagent Systems, 2008, pp. 331-338.

[22] J. Hao and H.-f. Leung, "Cuhkagent: An adaptive negotiation strategy for bilateral negotiations over multiple items," in Novel Insights in Agentbased Complex Automated Negotiation, ser. Studies in Computational Intelligence, I. Marsa-Maestre, M. A. Lopez-Carmona, T. Ito, M. Zhang, Q. Bai, and K. Fujita, Eds. Tokyo: Springer Japan, 2014, vol. 535, pp. 171-179.

[23] S. Chen, H. B. Ammar, K. Tuyls, and G. Weiss, "Optimizing complex automated negotiation using sparse pseudo-input gaussian processes," in Proceedings of the 2013 International Conference on Autonomous Agents and Multi-agent Systems, ser. AAMAS '13. Richland, SC: International Foundation for Autonomous Agents and Multiagent Systems, 2013, pp. 707-714

[24] Rosemarie Nagel, "Unraveling in guessing games: An experimental study," The American Economic Review, vol. 85, no. 5, pp. 1313-1326, 1995.

[25] R. Likert, "A technique for the measurement of attitudes." Archives of psychology, 1932.

[26] W. H. Kruskal and W. A. Wallis, "Use of ranks in one-criterion variance analysis," Journal of the American statistical Association, vol. 47, no. 260, pp. 583-621, 1952.

Simon Rothfuß Simon Rothfuß studied electrical engineering and information technology at the Karlsruhe Institute of Technology (KIT) and received the Master of Science in 2016. Today, he is a research associate at the Institute of Control Systems (IRS) at KIT and pursues his PhD degree. His main research interest is human-machine cooperative decision making.

Maximilian Wörner Maximilian Wörner studied electrical engineering and information technology at the Karlsruhe Institute of Technology (KIT) and the Technical University of Denmark (DTU). His main research interest is cooperative control regarding human-machine interaction. He received the Master of Science in 2021

Jairo Inga, Dr.-Ing. Jairo Inga received the B.Sc. (2011), M.Sc. (2014), and Dr.-Ing. (2020) in electrical engineering and information technology from the Karlsruhe Institute of Technology (KIT), Germany. He was a research assistant from 2015 to 2020 at the Institute of Control Systems at KIT and currently leads the Cooperative Systems research group at the same institute. His research interests include optimal control and game theory for modeling and identification of human behavior and the design of cooperative controllers in human-machine systems.

Andrea Kiesel, Prof. Dr. Andrea Kiesel studied psychology with a minor in artificial intelligence at the University of Würzburg. She received her $\mathrm{PhD}$ (2003) and her habilitation (2008) from University of Würzburg. Since 2015 she is head of the Cognition, Action and Sustainability Unit in the Institute of Psychology at the University of Freiburg. She is coordinator of a priority program on multitasking and involved in several research collaborations, e.g. in the excellence cluster on Living Materials Systems (LivMats) or a graduate school on Statistical Modelling in Psychology (SMiP). Currently, Andrea Kiesel is president of the European Society of Cognitive Psychology. Her research interests are human action control as well as technology acceptance.

Sören Hohmann, Prof. Dr.-Ing. Sören Hohmann studied electrical engineering at the Technische Universität Braunschweig, University of Karlsruhe and école nationale supérieure d'électricité et de mécanique Nancy. $\mathrm{He}$ received the diploma degree (1997) and $\mathrm{PhD}$ degree (2002) from University of Karlsruhe. Afterwards, until 2010 he worked in the industry for BMW, Munich, where his last position was head of the predevelopment and series developement of active safety systems. Today he is the head of the Institute of Control Systems at the Karlsruhe Institute of Technology, Germany as well as a director's board member of the research center for information technology (FZI), Karlsruhe. His research interests are cooperative control, alternative energies and system guarantees by design. 